\title{
Improvement in Pituitary Imaging After Targeted Therapy in Three Children with BRAF-Mutated Langerhans Cell Histiocytosis with Pituitary Involvement
}

This article was published in the following Dove Press journal: OncoTargets and Therapy

Ying Yang, (DD' Dong Wang,' $\mathrm{Na} \mathrm{Li},{ }^{2}$ Honghao Ma, ${ }^{1}$ Hongyun Lian,' Lei Cui, ${ }^{2}$ Qing Zhang, (iD ${ }^{2}$

Xiaoxi Zhao, ' Liping Zhang,' Yunze Zhao,' Chanjuan Wang, ${ }^{2}$ Li Zhang,' Tianyou Wang, ' Zhigang Li, ${ }^{2}$ Rui Zhang'

'Beijing Key Laboratory of Pediatric Hematology Oncology; National Key Discipline of Pediatrics; Key Laboratory of Major Diseases in Children, Ministry of Education; Hematology Oncology Center, Beijing Children's Hospital, Capital Medical University, National Center for Children's Health, Beijing 100045, People's Republic of China; ${ }^{2}$ Laboratory of Hematologic Diseases, Beijing Pediatric Research Institute, Beijing Children's Hospital, Capital Medical University, National Center for Children's Health, Beijing 100045 People's Republic of China
Correspondence: Rui Zhang; Tianyou Wang Beijing Children's Hospital, Capital Medical University, National Center for Children's Health, No. 56, Nanlishi Road, Xicheng District, Beijing, People's Republic of China Tel +86 010-13691156162

Fax +86 010-59617613

Email ruizh1973@I26.com;

wangtianyou@bch.com.cn

\begin{abstract}
Langerhans cell histiocytosis $(\mathrm{LCH})$ is a rare myeloid neoplasia in children. $B R A F$ mutations are associated with permanent diabetes insipidus (DI). The onset of clinical DI is always latent with imaging evidence of pituitary involvement. In this study, we reported three children with $B R A F$-mutated $\mathrm{LCH}$ with pituitary involvement who improved after targeted therapy (dabrafenib and trametinib). The results may indicate that pituitary involvement may be reversible in some patients with $\mathrm{LCH}$ if it is observed and treated before clinical DI. Targeted therapy may be an effective choice for LCH patients with early pituitary involvement who were irresponsive to first-line or second-line chemotherapy. However, a relapse after targeted therapy is completed in patients with persistently positive $\mathrm{cf} B R A F^{\mathrm{V} 600 \mathrm{E}}$ mutations is still a challenge that needs to be resolved. The timing of introducing targeted therapy, its appropriate duration and effective combinations with chemotherapy or other targeted drugs should be explored further.
\end{abstract}

Keywords: Langerhans cell histiocytosis, $B R A F$ mutation, pituitary, children, targeted therapy

\section{Introduction}

Langerhans cell histiocytosis $(\mathrm{LCH})$ is a rare myeloid neoplasia. The annual incidence of LCH in children younger than 15 years old is approximately 5 to 9 / $10^{6.1,2} \mathrm{LCH}$ is more common in children than in adults. The accumulation of pathologic Langerhans cells (LC) in the skin, bone, liver, spleen, bone marrow, central nervous system and so on causes a spectrum of clinical manifestations.

Diabetes insipidus (DI) is a clinical syndrome characterized by polyuria and polydipsia with diluted urine due to the inability to concentrate urine, ${ }^{3}$ which is a permanent sequela of LCH. It has been reported that the cumulative incidence of DI among patients with $\mathrm{LCH}$ is approximately $20 \%$ over 10 years $(13 / 70,5$ patients were treated with unspecified treatments, 7 patients with chemotherapy and 1 patient with radiotherapy). ${ }^{4}$ Some patients develop clinical DI several years after the initial diagnosis of $\mathrm{LCH}$; on the other hand, some patients present with DI several years prior to the diagnosis of $\mathrm{LCH}^{4}{ }^{4}$ The onset of clinical DI is always latent with imaging evidence of pituitary involvement, including infundibular thickening and the lack of a posterior pituitary bright spot on the T1-weighted 
magnetic resonance imaging (MRI) sequences. ${ }^{5}$ Longterm use of vasopressin replacement therapy is required once clinical DI occurs.

Pathologic LC is related to activation of the MAPK pathway (RAS/RAF/MEK/ERK). BRAF mutations are found in $50-60 \%$ of patients with $\mathrm{LCH}$, while $B R A F^{\mathrm{V} 600 \mathrm{E}}$ has been identified as comprising approximately $90 \%$ of $B R A F$ mutations and is associated with permanent damage, including pituitary injuries. ${ }^{6}$ Other $B R A F$ mutations, such as $B R A F^{\mathrm{V} 600 \mathrm{D}}$ and $B R A F^{\mathrm{V} 600 \mathrm{insDLAT}}$, have been reported. ${ }^{7}$

Patients with LCH with central nervous system (CNS) risk lesions appear to be associated with reactivation and are at a high risk of developing permanent sequelae, including neurodegeneration and DI. ${ }^{4}$ Therefore, before permanent sequelae occur in $\mathrm{LCH}$ patients with an affected pituitary, targeted therapy is expected to be effective. $^{6}$ Both BRAF kinase inhibitors (dabrafenib) and MEK inhibitors (trametinib) have been reported for use in the treatment of $\mathrm{LCH}$ patients. ${ }^{8,9}$ Here, we retrospectively reviewed three children with $B R A F$-mutated $\mathrm{LCH}$ with pituitary involvement who improved after targeted therapy, and we encourage clinicians to focus on the imaging findings of the pituitary and the efficacy of targeted therapy for $\mathrm{LCH}$.

\section{Subjects and Methods Patients}

We retrospectively analyzed all $\mathrm{LCH}$ patients who accepted targeted therapy with pituitary involvement (12 patients) and found three patients who had improved pituitary imaging after targeted therapy. The three patients we reported were diagnosed with $\mathrm{LCH}$ after histopathological examination with positive staining of CD1a and Langerin (CD207) of the skull (case 1), the shinbone (case 2) and a rash (case 3). BRAF mutations were detected in all of the patients from the biopsied tissues and/or circulation. They were followed up until July 1, 2020.

\section{Study Protocol}

All of the patients accepted first-line chemotherapy (vindesine + prednisone) after diagnosis and switched to targeted therapy due to a poor response or intolerance of the chemotherapy (Table 1). The dosages of dabrafenib and trametinib were, respectively, $2 \mathrm{mg} / \mathrm{kg}$ per treatment, once every 12 hours, and $0.5 \mathrm{mg}$ per treatment, once every day, administered orally. ${ }^{10}$ The general duration of the course of the targeted treatment was 6 months to 1 year, adjusted according to the disease assessment and patient tolerance of the drug. If the patients had disease progression, increased or persistently positive $\mathrm{cf} B R A F^{\mathrm{V} 600 \mathrm{E}}$ levels or severe adverse effects, targeted therapy could be discontinued.

After targeted therapy was completed, maintenance chemotherapy was given (6-mercaptopurine + vindesine + prednisone) for $0.5-1$ year. The affected lesions, pituitary MRI and adverse effects were evaluated after 1 month, 3 months, and every 3 months thereafter, according to the Histiocyte Society LCH Evaluation and Treatment Guidelines. ${ }^{11}$ During the course of targeted treatment, circulating cell-free $B R A F^{\mathrm{V} 600 \mathrm{E}}\left(\mathrm{cf} B R A F^{\mathrm{V} 600 \mathrm{E}}\right)$ in cases 1 and 3 were monitored (using the QX200TM Droplet Digital PCR System (Bio-Rad, Hercules, CA), and $\operatorname{cfBR} A F^{\mathrm{V} 600 \mathrm{E}}$ levels lower than $0.1 \%$ were regarded as negative). Currently, plasma $\mathrm{cf} B R A F^{\mathrm{V} 600 \mathrm{D}}$ cannot be monitored in our center.

\section{Examinations of Postpituitary Function}

The levels of thyroid-stimulating hormone (TSH), adrenocorticotropin (ACTH), gonadotropin hormone (GTH) and prolactin (PRL) were measured in cases 1 and 3 before the targeted therapy. Only TSH was measured in case 2 (the other hormones were not measured). We were unable to measure the levels of antidiuretic hormone (ADH) and oxytocin due to the limitations of the technical conditions in our center. We also measured the amount of fluid intake and urine volume per day, urine specific gravity and serum sodium of all patients before and after targeted treatment.

DI is defined as a clinical syndrome characterized by polyuria (in excess of $2 \mathrm{~L} / \mathrm{m}^{2} / 24 \mathrm{~h}$ ) and polydipsia with diluted urine (urine specific gravity less than 1.005). ${ }^{3}$ A water deprivation test was not performed for any of the patients because they had no clinical signs or symptoms of DI.

\section{Protocol of the Pituitary MRI}

Pituitary MRI was conducted at least twice and the images were evaluated by two experienced radiologists. MRI studies were performed using T1 weighted sequences in the sagittal and coronal planes (repetition time, $500 \mathrm{~ms}$; echo time, $8 \mathrm{~ms}$; flip angle, $90^{\circ}$ ) on a Philips Achieva TX 3.0T scanner. The slices were $3 \mathrm{~mm}$ thick, with a $130 \times 130 \mathrm{~mm}^{2}$ field of view and a $256 \times 256$ matrix. Imaging findings of the pituitary involvement included infundibular thickening, a mass lesion of the hypothalamic-pituitary axis and decreased or disappearing bright spots on the posterior pituitary on T1-weighted images (T1WI). ${ }^{5}$ The criteria for 


\begin{tabular}{|c|c|c|c|c|c|}
\hline \multicolumn{2}{|c|}{ 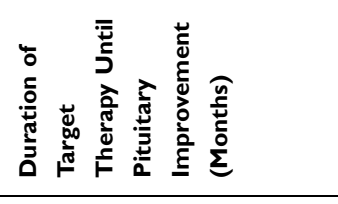 } & $m$ & - & $\mathrm{m}$ & 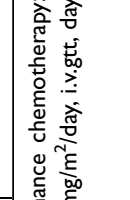 \\
\hline \multicolumn{2}{|l|}{ 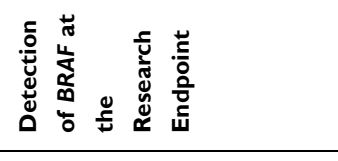 } & 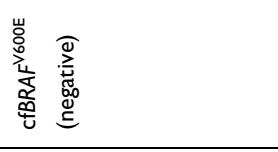 & 1 & 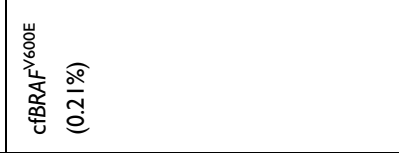 & 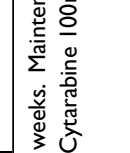 \\
\hline \multicolumn{2}{|l|}{ 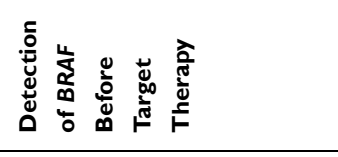 } & 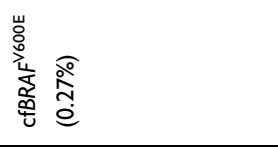 & 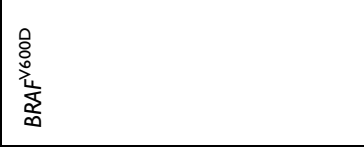 & 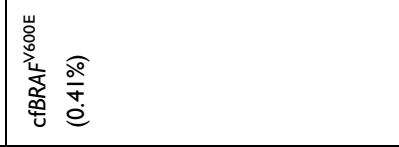 & 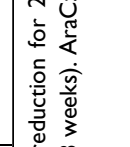 \\
\hline \multicolumn{2}{|l|}{ 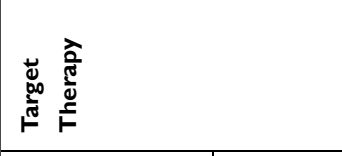 } & 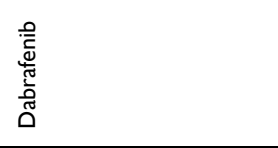 & 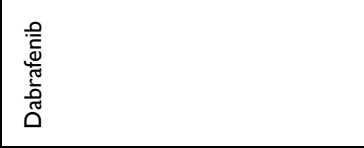 & 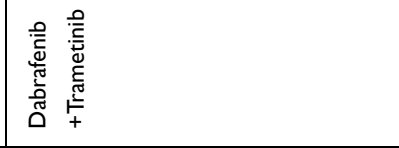 & 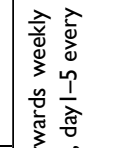 \\
\hline \multirow{2}{*}{ 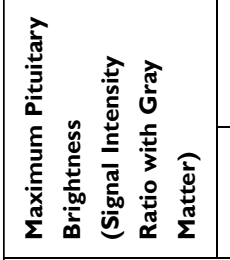 } & 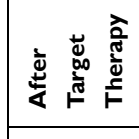 & 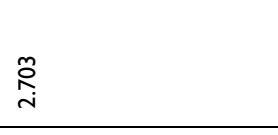 & $\frac{\stackrel{n}{N}}{2}$ & 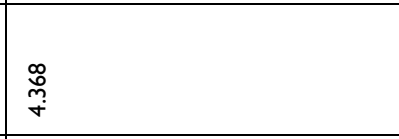 & 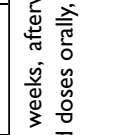 \\
\hline & 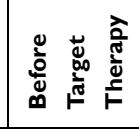 & $\stackrel{\infty}{\stackrel{\infty}{=}}$ & $\underline{E}$ & 商 & 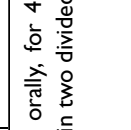 \\
\hline \multicolumn{2}{|l|}{ 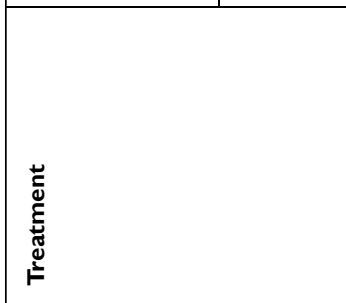 } & 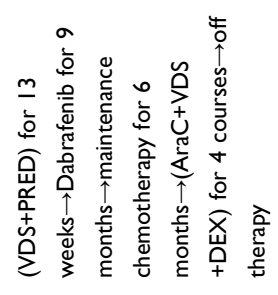 & 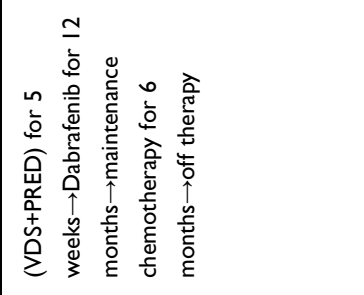 & 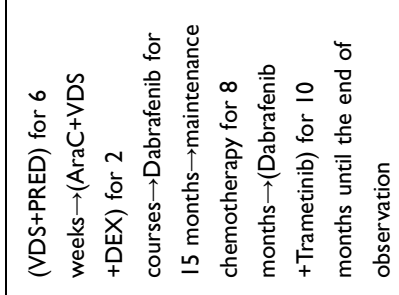 & 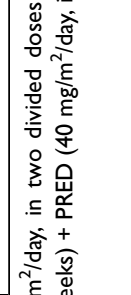 \\
\hline \multicolumn{2}{|l|}{ 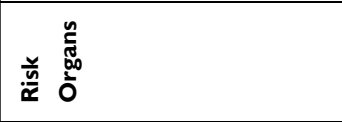 } & + & . & + & 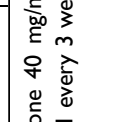 \\
\hline \multicolumn{2}{|l|}{ 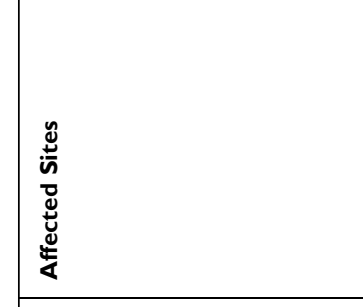 } & 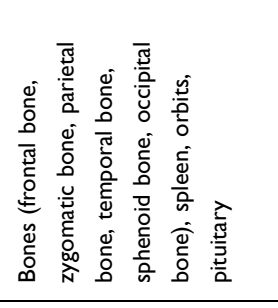 & 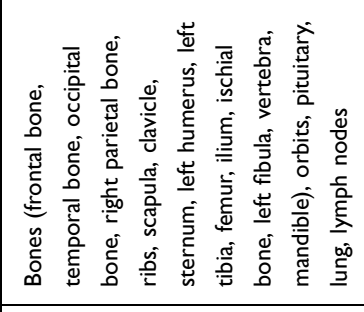 & 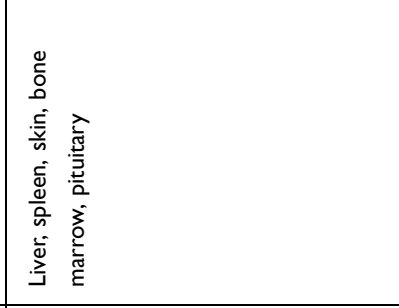 & 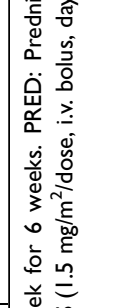 \\
\hline \multicolumn{2}{|l|}{$\overline{0}$} & z & $z$ & $z$ & \\
\hline \multicolumn{2}{|l|}{ 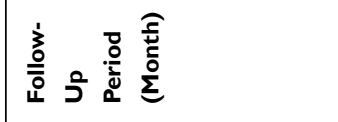 } & \pm & $\underline{\infty}$ & $\subseteq$ & 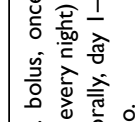 \\
\hline \multicolumn{2}{|l|}{ 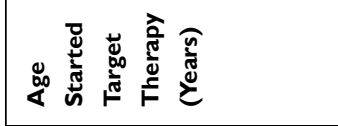 } & $\stackrel{\sim}{\check{m}}$ & $\hat{0}$ & $\stackrel{\circ}{\circ}$ & 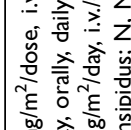 \\
\hline \multicolumn{2}{|l|}{ 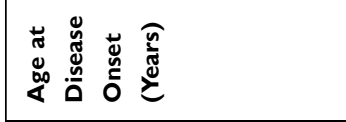 } & ส & นุ & ธี & 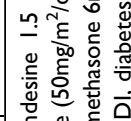 \\
\hline \multirow{2}{*}{\multicolumn{2}{|c|}{ ڤ̊ }} & $\frac{\frac{\omega}{\tilde{N}}}{\Sigma}$ & $\frac{\frac{0}{\sigma}}{\Sigma}$ & $\frac{\frac{0}{\tilde{I}}}{\Sigma}$ & \\
\hline & & 总 & 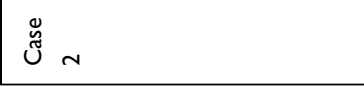 & 总 m & \\
\hline
\end{tabular}


an improvement in the pituitary findings on MRI included a reappearance of the missing spots or a normalization of the attenuated bright spots. Maximum pituitary brightness using the signal intensity ratio of the neighboring cerebral gray matter was the quantitative evaluation. No contrast was used.

\section{Results \\ Case I}

This patient was a 2.2-year-old boy diagnosed with multisystem-involved LCH (MS-LCH) with craniofacial involvement, ie, CNS-risk lesions. A lump on his head was the inaugural manifestation. The levels of $B R A F^{\mathrm{V} 600 \mathrm{E}}$ in skull biopsies and the plasma at diagnosis were $21.89 \%$ and $1.31 \%$, respectively. He had a normal urine volume (less than $2 \mathrm{~L} / \mathrm{m}^{2}$ / $24 \mathrm{~h}$ ) with a normal urine specific gravity and serum sodium. His levels of TSH, ACTH, GTH and PRL were also normal.

Because of a poor response to first-line chemotherapy (for 13 weeks) on his pituitary imaging (an absence of the normal posterior pituitary bright spot, Figure 1A; the other lesions were in a stable state), dabrafenib treatment was initiated at the age of 3.6, two weeks after completing the chemotherapy. After 3 months of dabrafenib treatment, the bright spot on the posterior pituitary on T1WI recurred with a higher signal intensity ratio compared with the gray matter (Figure 1B), and the other lesions also improved.

During targeted therapy, he experienced a mild skin allergic reaction, but the rash subsided after treatment with anti-allergic drugs. After the 9-month targeted therapy was completed, he was in an active disease (AD)-better state with a clear bright spot on the posterior pituitary on T1WI and then he was switched to second-line chemotherapy (cytarabine + vindesine + dexamethasone) because of being persistently positive for the $\operatorname{cf} B R A F^{\mathrm{V} 600 \mathrm{E}}$ mutation. Conversion to negative detection of $\mathrm{cf} B R A F^{\mathrm{V} 600 \mathrm{E}}$ was observed after 4 courses of second-line chemotherapy. At the end of follow-up, all therapy for LCH was terminated, and he was in an AD-better state without any clinical signs or symptoms of DI.

\section{Case 2}

In the second patient, MS-LCH was diagnosed at the age of 0.5 without polyuria or polydipsia. Fever was the main manifestation of the patient. Biopsied tissue from the involved shinbone showed $B R A F^{\mathrm{V} 600 \mathrm{D}}$. The amount of fluid intake and urine volume per day, urine specific gravity and serum sodium and TSH (the other hormones were not measured) were measured, which were all at normal levels.
Because of a poor response to first-line chemotherapy (for 5 weeks) on pituitary imaging (an absence of the normal posterior pituitary bright spot, Figure $1 \mathrm{C}$; the other lesions were in AD-better state), dabrafenib treatment was administered two weeks after completion of his chemotherapy. The brightness of the posterior pituitary on T1WI recovered after 1 month of dabrafenib treatment (Figure 1D). No target treatment-related side effects were observed. The duration of his dabrafenib treatment was 12 months. At the end of the observation period, the boy was followed up for 18 months without clinical DI, and no abnormality was observed on pituitary MRI. He has been off $\mathrm{LCH}$ treatment in an AD-better state.

\section{Case 3}

This patient was also a boy, and the age of his disease onset was 0.2 years. A rash was the initial presentation of the disease. His plasma $\operatorname{cfBR} A F^{\mathrm{V} 600 \mathrm{E}}$ was $3.29 \%$ at diagnosis, but no remaining biopsied rash sample could be acquired for $B R A F^{\mathrm{V} 600 \mathrm{E}}$ detection. Before treatment, he also had normal hormone levels, urine volume, urine specific gravity and serum sodium.

After the 6th week of first-line chemotherapy, he developed a progression of his skin lesions and was switched to second-line chemotherapy (cytarabine + vindesine + dexamethasone). However, after 2 courses of second-line chemotherapy, he had a serious infection followed by hemophagocytic lymphohistiocytosis and intolerance of chemotherapy with myelosuppression and gastrointestinal reactions. Therefore, dabrafenib was used alone for 15 months followed by maintenance chemotherapy.

At the 8th month of maintenance therapy, he had new involvement of the pituitary with decreased bright spots on the posterior pituitary on T1WI and infundibular thickening (Figure 1E; his other lesions were in an AD-stable state) and he began to take dabrafenib combined with trametinib.

After a 3-month combination targeted treatment, his pituitary brightness was higher with a recovered infundibulum. His circulating $\operatorname{cfBR} A F^{\mathrm{V} 600 \mathrm{E}}$ decreased from $0.41 \%$ to $0.24 \%$, and his other lesions also improved. At the end of the observation, the boy had taken combination targeted treatment for 10 months and did not experience $\mathrm{DI}$ in an AD-better state with clearer bright spots on the posterior pituitary on T1WI and had a recovered infundibulum (Figure 1F). His plasma $\operatorname{cf} B R A F^{\mathrm{V} 600 \mathrm{E}}$ had decreased to $0.21 \%$ at the research endpoint. 

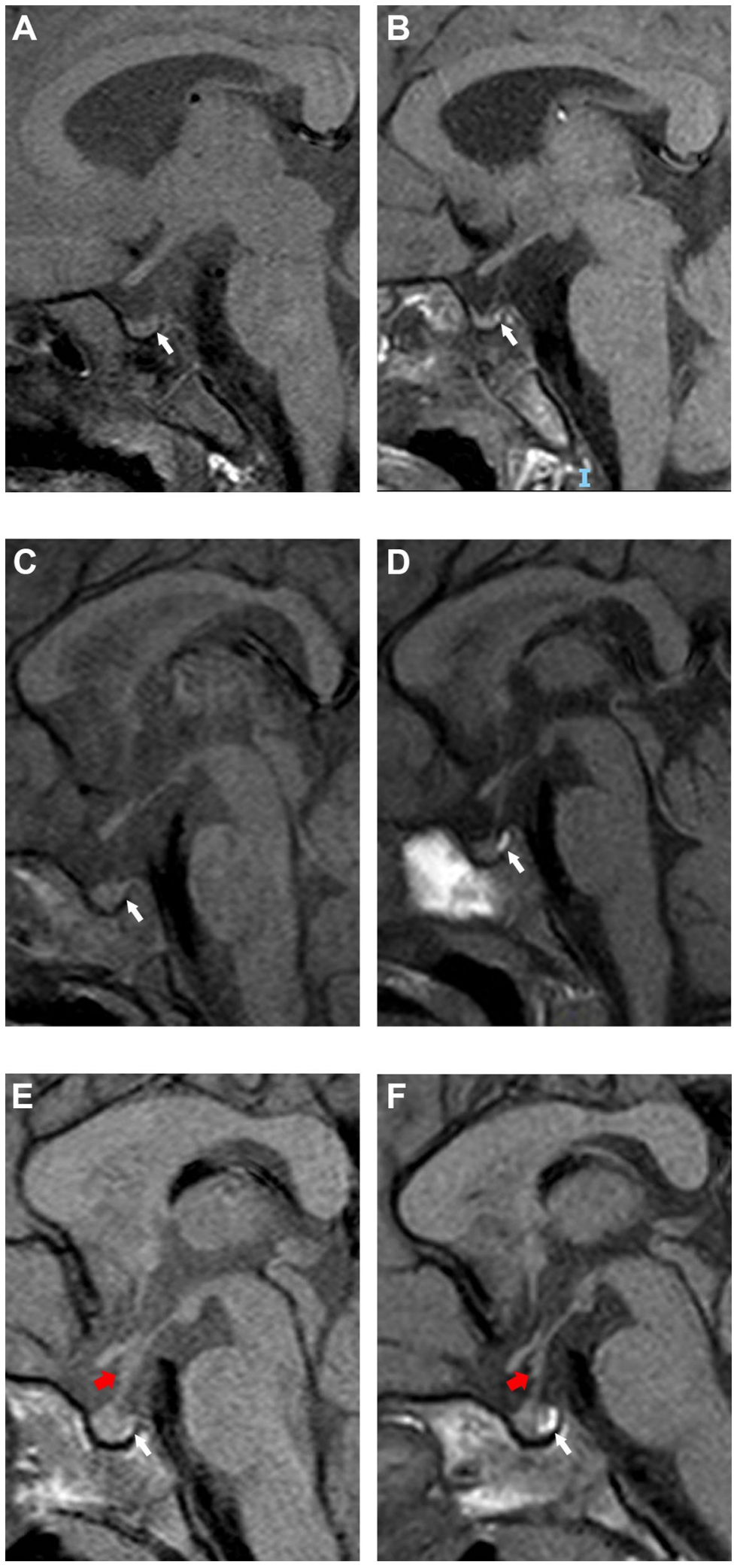

Figure I Pituitary MRI (sagittal plane) in the three patients reported in this study. In case I, (A) the bright-spot of posterior pituitary on TIWI was absent and (B) recurred after 3-month dabrafenib treatment (white arrowheads). In case 2, (C) the bright-spot of posterior pituitary was absent and (D) recurred after I-month dabrafenib treatment (white arrowheads). In case 3, (E) decreased bright-spot of posterior pituitary on TIWI (white arrowhead) and infundibular thickening (red arrowhead) were observed. (F) After I-month dabrafenib combined with trametinib treatment, the brightness was higher (white arrowhead) with recovered infundibulum (red arrowhead). 


\section{Discussion}

Due to the high risks of pituitary biopsies, pituitary imaging can be used instead to observe pituitary lesions even if patients do not have evident DI. The bright spot on the posterior pituitary on T1WI is related to vasopressin secretion granules or liposomes in pituitary cells. If pathologic LCs involve the pituitary gland, permanent DI occurs when there is a loss of $80 \%$ or more of the hypothalamic magnocellular neurons. ${ }^{12}$ Imaging findings of pituitary involvement may include infundibular thickening, a mass lesion of the hypothalamic-pituitary axis and decreased or absent bright spots on the posterior pituitary on T1WI. ${ }^{5}$

In our cases, all patients had BRAF mutations and specific imaging findings of pituitary involvement. The improvement of the pituitary imaging after targeted treatment can also support the early involvement of the pituitary before permanent DI occurs. Moreover, two of them had CNS-risk lesions that had a higher cumulative incidence of DI compared with patients without CNS-risk lesions reported in other studies. ${ }^{4,11}$

Previous studies have shown that DI is a permanent sequela, and the absence of the normal posterior pituitary bright spot is correlated with a loss of hypophyseal function and it may indicate an early stage of occult local tumors. ${ }^{3,13}$ Therefore, it is essential to initiate early treatment for patients with CNS-risk lesions and abnormal imaging of pituitary involvement before clinical DI occurs. As BRAF mutations are associated with pituitary involvement and resistance to first-line therapy, ${ }^{6,14}$ targeted therapy may improve pituitary lesions in $B R A F$-mutated $\mathrm{LCH}$ patients, based on the successful improvement in our cases.

Some LCH patients have persistently positive

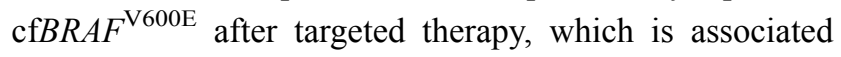
with a poor prognosis, especially relapses. ${ }^{14} \mathrm{We}$ administered second-line chemotherapy or maintenance chemotherapy after targeted treatment was completed to decrease the level of $\operatorname{cf} B R A F^{\mathrm{V} 600 \mathrm{E}}$ and reduce the recurrence rate. However, the timing of introducing targeted therapy has not achieved a consensus. Whether earlier administration rather than after first-line chemotherapy is more beneficial and the effectiveness and safety of longterm targeted treatment still need large-scale prospective studies.

\section{Conclusion}

In summary, these results may indicate that pituitary involvement may be reversible in some patients with LCH if it is observed and treated before overt clinical DI. Targeted therapy may be an effective choice for $\mathrm{LCH}$ patients with early pituitary involvement who are irresponsive to firstline or second-line chemotherapy. However, a relapse after targeted therapy is completed in patients with persistently positive $\mathrm{cfBRAF} F^{\mathrm{V} 600 \mathrm{E}}$ mutations is still a challenge that needs to be resolved. The timing of introducing targeted therapy, its appropriate duration and effective combinations with chemotherapy or other targeted drugs should be explored further.

\section{Abbreviations}

$\mathrm{AD}$, active disease; $\mathrm{ACTH}$, adrenocorticotropin; $\mathrm{ADH}$, antidiuretic hormone; CNS, central nervous system; DI, diabetes insipidus; GTH, gonadotropin hormone; $\mathrm{LCH}$, Langerhans cell histiocytosis; LC, Langerhans cells; MRI, magnetic resonance imaging; MS-LCH, multisystem-involved LCH; PRL, prolactin; T1WI, T1-weighted images; TSH, thyroid stimulating hormone.

\section{Data Sharing Statement}

The data that support the findings of this study are available on request from the corresponding authors.

\section{Ethics Approval}

Written informed consent (including informed consent to have the case details and any accompanying images published) was obtained from the parents or guardians of the children who served as subjects of the study. This study was conducted in accordance with the Declaration of Helsinki and approved by the Institutional Review Board (IRB) of Beijing Children's Hospital, Capital Medical University (Number: 2019-k-109). The regimen has been registered as a clinical trial (ChiCTR2000032844).

\section{Acknowledgments}

This work was supported by the Capital's Funds for Health Improvement and Research (No. 2020-2-2093 and 20202-1141), the Special Fund of The Pediatric Medical Coordinated Development Center of Beijing Hospitals Authority (No. XTZD20180201), the National Natural Science Foundation of China (No. 82070202), the National Science and Technology Key Projects (No. 2017ZX09304029004), Beihang University \& Capital Medical University Advanced Innovation Center for Big Data-Based Precision Medicine Plan (BHME-201912), and Funding for Reform and Development of Beijing Municipal health Commission (Genetic and immunological pathogenesis 
of pediatric histiocytosis and its guiding role in clinical diagnosis and treatment).

\section{Author Contributions}

All authors made a significant contribution to the work reported, whether that is in the conception, study design, execution, acquisition of data, analysis and interpretation, or in all these areas; took part in drafting, revising or critically reviewing the article; gave final approval of the version to be published; have agreed on the journal to which the article has been submitted; and agree to be accountable for all aspects of the work.

\section{Disclosure}

The authors declare no conflicts of interest in this work.

\section{References}

1. Emile JF, Abla O, Fraitag S, et al. Revised classification of histiocytoses and neoplasms of the macrophage-dendritic cell lineages. Blood. 2016;127(22):2672-2681. doi:10.1182/blood-2016-01-690636

2. Allen CE, Merad M, McClain KL, Longo DL. Langerhans-cell histiocytosis. N Engl J Med. 2018;379(9):856-868. doi:10.1056/ NEJMra1607548

3. Di Iorgi N, Napoli F, Allegri AE, et al. Diabetes insipidus-diagnosis and management. Horm Res Paediatr. 2012;77(2):69-84. doi:10.1159/ 000336333

4. Chow TW, Leung WK, Cheng FWT, et al. Late outcomes in children with Langerhans cell histiocytosis. Arch Dis Child. 2017;102 (9):830-835. doi:10.1136/archdischild-2016-312185
5. Kaltsas GA, Powles TB, Evanson J, et al. Hypothalamo-pituitary abnormalities in adult patients with Langerhans cell histiocytosis: clinical, endocrinological, and radiological features and response to treatment. J Clin Endocrinol Metab. 2000;85(4):1370-1376. doi:10. 1210/jcem.85.4.6501

6. Heritier S, Emile JF, Barkaoui MA, et al. BRAF mutation correlates with high-risk Langerhans cell histiocytosis and increased resistance to first-line therapy. $J$ Clin Oncol. 2016;34(25):3023-3030. doi:10.1200/JCO.2015.65.9508

7. Durham BH. Molecular characterization of the histiocytoses: neoplasia of dendritic cells and macrophages. Semin Cell Dev Biol. 2019;86:62-76. doi:10.1016/j.semcdb.2018.03.002

8. Kieran MW, Geoerger B, Dunkel IJ, et al. A phase I and pharmacokinetic study of oral dabrafenib in children and adolescent patients with recurrent or refractory BRAF V600 mutation-positive solid tumors. Clin Cancer Res. 2019;25(24):7294-7302. doi:10.1158/ 1078-0432.CCR-17-3572

9. Arico M. Langerhans cell histiocytosis in children: from the bench to bedside for an updated therapy. $B r J$ Haematol. 2016;173 (5):663-670. doi:10.1111/bjh.13955

10. Yang Y, Wang D, Cui L, et al. Effectiveness and safety of dabrafenib in the treatment of 20 Chinese children with $B R A F^{\mathrm{V} 600 \mathrm{E}}$-mutated LCH. Cancer Res Treat. 2020. doi:10.4143/crt.2020.769

11. Histiocyte society LCH evaluation and treatment guidelines [Internet]; 2009. Available from: https://histiocytesociety.org/docu ment.doc?id=290. Accessed November 19, 2020.

12. Bellastella A, Bizzarro A, Colella C, et al. Subclinical diabetes insipidus. Best Pract Res Clin Endocrinol Metab. 2012;26 (4):471-483. doi:10.1016/j.beem.2011.11.008

13. Adams NC, Farrell TP, O'Shea A, et al. Neuroimaging of central diabetes insipidus-when, how and findings. Neuroradiology. 2018;60 (10):995-1012.

14. Cui L, Zhang L, Ma HH, et al. Circulating cell-free BRAF V600E during chemotherapy is associated with prognosis of children with Langerhans cell histiocytosis. Haematologica. 2020;105(9):e444447. doi:10.3324/haematol.2019.229187
OncoTargets and Therapy

\section{Publish your work in this journal}

OncoTargets and Therapy is an international, peer-reviewed, open access journal focusing on the pathological basis of all cancers, potential targets for therapy and treatment protocols employed to improve the management of cancer patients. The journal also focuses on the impact of management programs and new therapeutic agents and protocols on patient perspectives such as quality of life, adherence and satisfaction. The manuscript management system is completely online and includes a very quick and fair peer-review system, which is all easy to use. Visit http://www.dovepress.com/ testimonials.php to read real quotes from published authors. 